\title{
Oral and Intravenous Iron Therapy Differentially Alter The on- and Off-Tumour Microbiota in Anaemic Colorectal Cancer Patients.
}

\section{Oliver Phipps ( $\sim$ O.phipps@wlv.ac.uk)}

RIHS: University of Wolverhampton Research Institute in Healthcare Science https://orcid.org/00000003-4588-4869

\section{Hafid Omar Al-Hassi}

RIHS: University of Wolverhampton Research Institute in Healthcare Science

\section{Mohammed Nabil Quraishi \\ University of Birmingham}

\section{Edward A Dickson}

NIHR Nottingham Biomedical Research Centre

\section{Jonathan Segal}

St Mark's Hospital Foundation

\section{Helen Steed}

Royal Wolverhampton Hospitals NHS Trust

\section{Aditi Kumar}

Royal Wolverhampton Hospitals NHS Trust

\section{Austin G Acheson}

NIHR Nottingham Biomedical Research Centre

\section{Andrew D Beggs}

University of Birmingham

\section{Matthew J Brookes}

Royal Wolverhampton Hospitals NHS Trust

\section{Research}

Keywords: Iron deficiency anaemia, Anaemic patients, Oral iron, anti-inflammatory, tumour protective pathways, off-tumour microbiota

Posted Date: January 14th, 2021

DOI: https://doi.org/10.21203/rs.3.rs-143378/v1 
License: (c) (i) This work is licensed under a Creative Commons Attribution 4.0 International License. Read Full License 


\section{Abstract}

Background Iron deficiency anaemia is a common complication of colorectal cancer and may require iron therapy. Oral iron can increase iron available to gut bacteria and may alter the colonic microbiota. We performed an intervention study to compare oral and intravenous iron therapy on the colonic tumourassociated (on-tumour) and paired non-tumour-associated adjacent (off-tumour) microbiota. Anaemic patients with colorectal adenocarcinoma received either oral ferrous sulphate $(n=16)$ or intravenous ferric carboxymaltose $(n=24)$. On- and off-tumour biopsies were obtained post-surgery and microbial profiling was performed using $16 \mathrm{~S}$ ribosomal RNA analysis.

Results Off-tumour $\mathrm{a}$ - and $\beta$-diversity were significantly different between iron treatment groups. No differences in on-tumour diversity were observed. Off-tumour microbiota of oral iron-treated patients shows higher abundances of the orders Clostridiales, Cytophagales and Anaeroplasmatales compared to intravenous iron-treated patients. The on-tumour microbiota was enriched with the orders Lactobacillales and Alteromonadales in the oral and intravenous iron groups, respectively. The on- and off-tumour microbiota associated with intravenous iron-treated patients infers increased abundances of enzymes involved in iron sequestration and anti-inflammatory/oncogenic metabolite production, compared to oral iron-treated patients. Paired on- and off-tumour microbiota show large taxonomic differences in intravenous iron-treated patients and limited differences in oral iron-treated patients.

Conclusion Oral iron shows a large shift in the off-tumour microbiota, but a more limited change in ontumour microbiota. The on- and off-tumour microbiota in intravenous iron-treated patients infers a microbiota associated with anti-inflammatory and tumour protective pathways. Suggesting intravenous iron may be a more appropriate therapy to limit adverse microbial outcomes, compared to oral iron.

Trial registration: NCT01701310. Registered 21 March 2012, https://clinicaltrials.gov/ct2/show/NCT01701310

\section{Introduction}

Perturbations in gut bacterial populations are characteristic of colorectal cancer, with the presence of pathogenic indigenous bacterial species at the expense of protective probiotic species [1]. Driver bacteria are involved in the initiation of colorectal cancer. Which are gradually outcompeted by opportunistic passenger bacteria, which have a selective advantage within the newly defined tumour-

microenvironment. These passenger bacteria can potentially either promote or hinder tumour progression dependent on whether pathogenic or probiotic bacterial populations flourish [2]. Hence, external factors that can influence gut bacterial growth, such as iron supplementation in anaemic colorectal cancer patients, can potentially support colorectal cancer progression dependent on the bacterial populations they promote $[2,3]$.

Iron is essential for the growth and development of the vast majority of gut bacteria, with pathogenic bacteria tending to have heightened iron acquisition mechanisms [4]. Hence, in anaemic colorectal cancer 
patients the use of oral iron supplementation has the potential to alter bacterial populations of the colorectal tumour-associated (on-tumour) microbiota, as well as the non-tumour-associated mucosal (offtumour) microbiota $[5,6]$. This may suggest that a parenteral route of iron administration may be more beneficial to treat anaemia, without increasing iron availability to colonic bacteria. Initial data from the intravenous iron in colorectal cancer associated anaemia (IVICA) trial suggests that intravenous iron may be more beneficial than oral iron in treating pre-operative anaemia [7]. However, results from the preoperative intravenous iron to treat anaemia before major abdominal surgery (PREVENTT) trial has put doubt over the efficacy of intravenous iron pre-operatively [8]. Hence, which is the most beneficial treatment of anaemia pre-operatively, oral or intravenous iron, remains uncertain. The purpose of this study is to determine if patients treated with oral iron have a more procarcinogenic microbiota compared to those treated with intravenous iron.

Conflicting evidence exists regarding the effect of iron supplementation in murine studies of gut bacterial diversity $[9,10]$. However, to our knowledge a comparison of the gut microbiota following oral and intravenous iron therapy in human studies of colorectal cancer has yet to be explored. This pilot intervention study aims to compare the outcomes of oral and intravenous iron therapy on the on- and offtumour microbiota in colorectal cancer patients with iron deficiency anaemia.

\section{Methods}

\section{Study population}

From the IVICA trial a total of 40 anaemic patients with non-metastatic colorectal adenocarcinoma were randomised to receive either oral ferrous sulphate $200 \mathrm{mg}$ twice a day $(n=16)$ or intravenous iron (ferric carboxymaltose - Ferinject ${ }^{\mathrm{T}}{ }^{\mathrm{m}}$; Vifor Pharma, Glattbrugg, Switzerland) dosed by weight and haemoglobin in accordance with the summary of product characteristics $(n=24)$ [7]. Treatment was administered at least 2 weeks pre-operatively and anaemia was defined as having a haemoglobin level $10 \mathrm{~g} / \mathrm{l}$ below the sexspecific World Health Organisation definition (women $\leq 120 \mathrm{~g} / \mathrm{l}$, men $\leq 130 \mathrm{~g} / \mathrm{l}$ ). Duration of iron treatment and inclusion haemoglobin for treatment groups are presented in the patient demographics in table 1. To limit the inclusion of non-iron deficient anaemic individuals, those with pre-existing haematological disease, renal failure or undergoing current chemotherapy were not eligible for the trial. Colorectal tumour biopsies and paired tumour-adjacent colonic mucosal tissue biopsies were obtained post-surgery.

\section{DNA extraction and 16S rRNA amplicon sequencing}

Microbial DNA was extracted from colorectal tumour biopsies and paired tumour-adjacent mucosal colonic tissue biopsies using a modified protocol of Qiagen All Prep DNA/RNA Mini Kit (Qiagen, Hilden, Germany). Biopsies were mechanically lysed using $5 \mathrm{~mm}$ steel bead (Qiagen) and $0.1 \mathrm{~mm}$ Zirconia/Silica beads (Strateck, Suffolk, UK) with a TissueLyser (Qiagen), followed by enzymatic and heat lysis. Extracted microbial DNA was used for $16 \mathrm{~S}$ ribosomal RNA (rRNA) gene amplification and sequencing to determine the mucosal-adherent microbiota according to the Earth Microbiome project protocol [11]. 
Using primers targeted to the V4 region (515F-806R), the 16S rRNA genes were amplified in technical triplicates. This was performed using a single-step, single-indexed polymerase chain reaction (PCR). DNA extraction and 16S rRNA gene PCR were both performed in batch with appropriate multiple-reagent based negative controls. Paired-end sequencing ( $2 \times 250$ base pairs) was completed in a single batch using the Illumina MiSeq platform (Illumina, San Diego, USA).

\section{Statistical analysis}

Microbial analysis was performed using the Quantitative Insight Into Microbial Ecology 2 (QIIME2) pipeline [12]. Bacterial taxonomy was assigned using the Silva-132-99\% operational taxonomic units (OTUs) database [13]. Intra-sample comparisons (a-diversity) were assessed using Mann-Whitney $U$ test comparing variation in Abundance-based Coverage Estimator (ACE), Chao1, Shannon diversity index, Faith's phylogenetic diversity and Observed OTUs. Inter-sample comparisons ( $\beta$-diversity) were assessed using permutational multivariate analysis of variance (PERMANOVA) comparing Bray-Curtis dissimilarity and Jaccard similarity using principal coordinate analysis (PCOA). a-diversity indices and PCoA were plotted using the R package "ggplot" [14]. Comparison of relative abundances of taxa between treatment groups and sample types were performed using a linear discriminant analysis (LDA) effect size (LEfSe), taxa with an LDA score greater than 2 with a $p$-value $\leq 0.05$ were considered statistically significant [15]. Predictive functional profiles from microbial populations were derived using Phylogenetic Investigation of Communities by Reconstruction of Unobserved States 2 (PICRUSt2), using enzyme classification pathway analysis. Differences in predictive enzyme abundances were assessed using Statistical Analysis of Metagenomic Profiles (STAMP). Corrected q-values were calculated following multiple testing correction using Storey false discovery rate. q-values $\leq 0.05$ between treatment groups were considered statistically significant [16-18].

\section{Results}

\section{On- and off-tumour bacterial diversity following iron therapy}

A total of 7.9 million reads ( 109,777 reads/sample) and 2,367 features were obtained following quality control, with a sampling depth of 8,000 reads/sample. Comparison of a-diversity metrics shows that patients treated with oral iron have a significantly higher bacterial diversity (Shannon Diversity and Faith's Phylogenetic Diversity), richness (Observed OTUs), and abundance (Chao1 and ACE) within their offtumour microbiota, relative to those treated with intravenous iron therapy (Figure $1 ; p<0.05$ ). Consistent with this Jaccard similarity and Bray-Curtis dissimilarity assessed $\beta$-diversity between treatment groups and show that within the off-tumour microbiota, oral and intravenous iron form significantly different bacterial community clusters (Figure 2; $p<0.05$ ).

The on-tumour microbiota of these patients shows no differences in a-diversity between iron treatment groups (Figure $1 ; n s$ ). Along with this, no significant differences in $\beta$-diversity were observed (Figure 2 : $n s)$. Taken together this suggests that oral iron and intravenous iron-treated patients show significantly 
different bacterial diversity in their tumour-adjacent microbiota. However, the tumour-associated microbiota shows a consistent bacterial diversity between iron treatments.

\section{Oral and intravenous iron-treated patients show differing bacterial communities}

Differences in microbial populations between oral and intravenous iron-treated patients were assessed using LEfSe to determine bacterial taxa that are significantly enriched between treatments (Figure 3 ). The off-tumour microbiota of oral iron-treated patients shows a greater abundance of the Clostridia class, Clostridiales, Anaeroplasmatales and Cytophagales orders, Lactobacillaceae and Anaeroplasmataceae families and Lactobacillus, Agathobacter, Coprococcuss 3, Eubacterium eligens groups, Eubacterium xylanophilum group, Lachnospiraceae ND3007 group, Ruminococcaceae NK4A214 group, Ruminococcus 1, Ruminococcus 2 and Anaeroplasma genera. Whereas in the intravenous iron group there was higher abundance of Lachnospiraceae NK4A136 group genus (Figure 3a, b).

The on-tumour microbiota in oral iron-treated patients shows a greater abundance of the Bacilli class, Lactobacillales order and Prevotella 7, Butyrivibrio, Coprococcus 1, Hungatella, Lachnospiraceae ND3007 group, Eubacterium ventriosum group, Ruminococcaceae NK4A214 group and Pleomorphomonas genera. Whereas the on-tumour microbiota of intravenous iron-treated patients show higher abundance of the Alteromonadales order and Alloprevotella and Enhydrobactergenera (Figure 3c, d). These results suggest that the on- and off-tumour microbiota show differential responses to iron therapy. With oral iron leading to a more prominent change in bacterial taxa in the tumour-adjacent compared to the tumourassociated microbiota.

\section{Differential predictive enzymatic pathways between iron treatment groups}

In order to infer the metagenomic pathways associated with microbial profiles, we performed a PICRUSt2 metagenomic analysis using predicted enzyme classification abundances based on 16S rRNA bacterial populations. These show a large difference in predicted microbial enzymes between iron treatments in the off-tumour microbiota (Figure 4a), while fewer differences in the on-tumour microbiota were observed (Figure 4b). The off-tumour microbiota is associated with increased abundance of iron-related enzymes, Bacterial non-haem ferritin and Ferric-chelate reductase, in intravenous iron-treated patients compared to oral iron. Along with this, the off-tumour microbiota of intravenous iron-treated patients shows increased abundance of enzymes involved in the production of anti-inflammatory metabolites, including Lactaldehyde dehydrogenase and 2-iminobutanoate/2-iminopropanoate deaminase (Figure 4a).

The on-tumour microbiota is associated with increased abundance of enzymes involved in antiinflammatory and colorectal cancer protective metabolite production, including Cellulose synthase and Nsulfoglucosamine sulfohydrolase, in intravenous iron-treated patients (Figure 4b). Overall, the predictive metagenomic results suggest that the on- and off-tumour microbiota profiles associated with intravenous iron-treated patients are involved in regulating iron metabolism and production of metabolites involved in inhibiting intestinal inflammatory and colorectal cancer, compared to oral iron-treated patients. 


\section{Paired on- and off-tumour microbiota show varying microbial communities following oral and intravenous iron therapy}

As the tumour-associated and tumour-adjacent microbiota show differing outcomes following iron therapy in respect to bacterial taxa, we aimed to assess if the method of iron administration led to changes between paired on- and off-tumour microbiota (Figure 5a). Patients treated with oral iron show their off-tumour microbiota being enriched with the Bacteroidaceae family and Bacteroides genus, while their on-tumour microbiota shows a higher abundance of Nocardiodaceae, Intrasporangiaceae and Brevibacteriaceae families and Prevotella 9, Nocardioides, Kocuria, Brevibacterium, Veillonella and Catenibacterium genera (Figure $5 c, d$ ).

In contrast, patients treated with intravenous iron show their off-tumour microbiota being enriched with the Firmicutes phylum and Clostridia class, along with a greater abundance of the Clostridiales and Sphingomonadales orders, Sphingomonadaceae family and Paraprevotella genus. Whereas the ontumour microbiota of patients treated with intravenous iron shows a higher abundance of the Epsilonbacteraeota phylum, Campylobacteria class, Campylobacterales order, Campylobacteraceae, Propionibacteriaceae and Porphyromonadaceae families and Campylobacter, Porphyromonas and Cutibacterium genera (Figure 5a, b). These results suggest that patients treated with oral iron have a more consistent tumour-associated and tumour-adjacent microbiota, showing only small changes at lower taxonomic levels. Whereas patients treated with intravenous iron show a much greater difference between their tumour-associated and tumour-adjacent microbiota, with major differences at the phylum, class, and order levels.

\section{Discussion}

A high incidence of colorectal cancer-associated iron deficiency leads to the requirement of therapeutic iron to correct anaemia, with oral iron being the standard treatment due to low cost and convenience [19, 20]. However, oral iron is associated with gastrointestinal side-effects and can increase colonic iron concentration, which can contribute to oncogenic signalling and colitis [21-23]. However, to our knowledge this is the first study to investigate microbial outcomes of oral iron supplementation in anaemic colorectal cancer patients.

This study shows that the on- and off-tumour microbiota shows differential outcomes following oral and intravenous iron supplementation, demonstrating differences in a- and $\beta$-diversity, bacterial taxa, and predictive metagenomics. Off-tumour bacterial diversity is significantly different between oral and intravenous iron treatments, supporting a defined off-tumour microbial profile following each therapy. This is consistent with large differences in bacterial taxa, showing greater abundance of Clostridia class, Anaeroplasmatales, Clostridiales and Cytophagales orders and Lactobacillaceae and Anaeroplasmataceae families in oral iron-treated patients. In contrast, the on-tumour microbiota is more consistent between iron treatments, showing no difference in diversity metrics. Potentially pre-existing tumour microbial alterations may prevent oral iron from leading to major shifts in bacterial populations. 
However, differences in bacterial taxa are present between treatment groups, including a higher abundance of Bacilli class and Lactobacillales order in the oral iron group and higher abundance of Alteromonadales order in the intravenous iron group.

On- and off-tumour bacterial genera that are more abundant in oral iron-treated patients include Coprococcus, Prevotella and Ruminococcus (Figure 3a, b). A previous study by Flemer et al[24] identified colorectal cancer enriched microbiota, showing that Coprococcus, Prevotella and Ruminococcus were all colorectal cancer enriched genera [24]. Furthermore, a study by Kim et a/[25] found that the genera Ruminococcus 2 and Ruminococcaceae NK4A214 group, which were more abundant in oral iron-treated patients (Figure $3 a, b)$, were enriched in colorectal cancer patient. Whereas the genus Lachnospiraceae NK4A136 group, which was more abundant in intravenous iron-treated patients (Figure 3a), was depleted in colorectal cancer patients [25]. Comparing our results to these studies suggests that oral iron may increases the abundance of colorectal cancer enriched genera, whereas intravenous iron is associated with colorectal cancer depleted genera. However, what is not stated in these studies is whether these are driver or passenger bacteria in colorectal cancer. Potentially beneficial bacterial genera were differentially enriched between iron treatments. Potentially probiotic Lactobacillus were more abundant in oral iron (Figure 3a), whereas anti-inflammatory acetic and butyric acid-producing Alloprevotella and Lachnospiraceae NK4A136 group were more abundant in intravenous iron-treated patients (Figure 3a, b) [26-28]. Collectively the results of our study are consistent with previous findings by Lee et al[29], showing the faecal microbiota of oral and intravenous iron-treated inflammatory bowel disease patients had differential bacterial communities. With the authors suggesting intravenous iron may be more beneficial for treating anaemic Crohn's disease patients, in order to limit microbial perturbations [29].

Predictive metagenomics shows the off-tumour microbiota of intravenous iron-treated patients has greater abundance of bacterial enzymes involved in iron metabolism, compared to oral iron (Figure 4). Ferric-chelate reductase is a bacterial enzyme that catalases the reduction of siderophore-bound ferriciron to release ferrous-iron [30]. Bacterial non-haem ferritin is a storage protein that binds ferrous-iron keeping it inert intracellularly [31]. Collectively these can contribute to bacterial sequestration of colonic luminal iron, hence not biologically available to contribute to colonic inflammation and tumour initiation [32]. The off-tumour microbiota of intravenous iron-treated patients also shows an increased abundance of Lactaldehyde dehydrogenase, which is involved in the production of anti-inflammatory lactate and pyruvate [33-35]. Likewise, 2-iminobutanoate/2-iminopropanoate deaminase is involved in isoleucine biosynthesis, which can limit intestinal inflammation and inhibit colorectal cancer metastasis [36, 37]. The on-tumour microbiota of intravenous iron-treated patients shows increased abundance of Cellulose synthase. This bacterial enzyme produces cellulose, which can inhibit colonic inflammation through modulating lipid metabolism and inactivating secondary bile acids [38, 39]. N-sulfoglucosamine sulfohydrolase produces glucosamine, which is anti-inflammatory and colorectal cancer protective [40, 41]. Collectively this infers that the on- and off-tumour microbiota in intravenous iron-treated patients is associated with iron sequestration, along with anti-inflammatory and tumour protective metabolite production, compared to oral iron-treated patients. It is remains to be investigated whether the PICRUSt2 results are functional to further confirm the outcome of this study. 
Paired on- and off-tumour microbiota show differentially enriched bacterial taxa in the oral and intravenous iron cohorts. The intravenous iron group shows substantial differences between the on- and off-tumour microbiota at the phylum level. With the off-tumour microbiota being enriched with Firmicutes and the on-tumour microbiota with Epsilonbacteraeota. In contrast, patients treated with oral iron show few differences, primarily at the family and genus level. This suggests that oral iron-treated patients have a more consistent on- and off-tumour microbiota, compared to more diverse in intravenous iron-treated patients.

This pilot study provides novel insights into microbial outcomes of iron therapy in colorectal cancer, suggesting oral iron may be a more deleterious microbial-altering iron therapy. Whereas intravenous iron therapy may be more appropriate in this cohort of anaemic patients. Despite the relatively small sample size of this study, significant differences were observed between iron treatment groups, which can form the foundation for further large-scale explorative studies. These studies could account for confounding variables, such as diet, that have the potential to lead to differential microbial populations, as well as including comparator colonic mucosal microbiota from healthy controls or pre-iron treatment. The relatively small window of therapeutic intervention in this study may show differential microbial outcomes compared to longer-term iron therapy. Nevertheless, this period relevantly reflects the common clinical intervention in anaemic colorectal cancer patients prior to surgery. Furthermore, this study could be further validated through a more comprehensive study of bacterial metabolomics following iron therapy, as well as addressing the clinical relevance of differential microbial communities on long term outcomes following surgery in these patients.

\section{Declarations}

Ethics approval and consent to participate Ethical approval for the IVICA trial was granted by the National Research and Ethics Service (East Midlands - Nottingham 2 Research Ethics Committee, 11/EM/0237). The study was registered with Clinical Trials.Gov (NCT01701310) and the Medicines and Healthcare Products Regulatory Agency (2011-002185-21). The study was undertaken in line with the Declaration of Helsinki.

Consent for publication Not applicable.

Availability of data and material The datasets generated during and/or analysed during the current study are available from the corresponding author on reasonable request.

Competing interests MJB's research department has received grant support from Tillots Pharma and Vifor Pharma (Switzerland). MJB has received honoraria and travel support for consulting or lecturing from Vifor Pharma, Tillots Pharma, and Abbvie. The funders had no role in the writing of the manuscript.

Funding The Ferinject ${ }^{\text {TM }}$ used in the original IVICA trial was donated to all study centres, except Nottingham University Hospitals NHS Trust, by Vifor Pharma (Glattbrugg, Switzerland). The study represents independent research funded by the National Institute for Health Research (NIHR) Research for 
Patient Benefit (RfPB) programme (grant number PB-PG-0110-21041). The views expressed are those of the authors and not necessarily those of the National Health Service, the NIHR or the Department of Health.

Authors' contributions Study design: HOA, HS, AGA, ADB and MJB. Data acquisition: OP, HOA and MNQ. Statistical analysis: OP and MNQ. Data interpretation and manuscript writing: OP, HOA, MNQ, EAD, JS, HS, AK, AGA, ADB, MJB.

Acknowledgements Not applicable.

\section{References}

1. Khan I, Ullah N, Zha L, Bai Y, Khan A, Zhao T, et al: Alteration of Gut Microbiota in Inflammatory Bowel Disease (IBD): Cause or Consequence? IBD Treatment Targeting the Gut Microbiome. Pathogens. 2019;8(3).

2. Tjalsma H, Boleij A, Marchesi JR, Dutilh BE: A bacterial driver-passenger model for colorectal cancer: beyond the usual suspects. Nature reviews. Microbiology. 2012;10(8):575-582.

3. Song M, Chan AT, Sun J: Influence of the Gut Microbiome, Diet, and Environment on Risk of Colorectal Cancer. Gastroenterology. 2020;158(2):322-340.

4. Cassat JE, Skaar EP: Iron in Infection and Immunity. Cell Host Microbe. 2013;13(5):509-519.

5. Phipps O, Al-Hassi HO, Quraishi MN, Kumar A, Brookes MJ: Influence of Iron on the Gut Microbiota in Colorectal Cancer. Nutrients. 2020;12(9).

6. Flemer B, Herlihy M, O'Riordain M, Shanahan F, O'Toole PW: Tumour-associated and non-tumourassociated microbiota: Addendum. Gut Microbes. 2018;9(4):369-373.

7. Keeler BD, Simpson JA, Ng O, Padmanabhan H, Brookes MJ, Acheson AG, et al: Randomized clinical trial of preoperative oral versus intravenous iron in anaemic patients with colorectal cancer. $\mathrm{Br} \mathrm{J}$ Surg. 2017;104(3):214-221.

8. Richards T, Baikady RR, Clevenger B, Butcher A, Abeysiri S, Chau M, et al: Preoperative intravenous iron to treat anaemia before major abdominal surgery (PREVENTT): a randomised, double-blind, controlled trial. The Lancet (British edition). 2020;396(10259):1353-1361.

9. Mahalhal A, Williams JM, Johnson S, Ellaby N, Duckworth CA, Burkitt MD, et al: Oral iron exacerbates colitis and influences the intestinal microbiome. PLoS ONE. 2018;13(10):e0202460.

10. Liu B, Pan X, Liu Z, Han M, Xu G, Dai X, et al: Fecal microbiota as a noninvasive biomarker to predict the tissue iron accumulation in intestine epithelial cells and liver. FASEB J. 2020;34(2):3006-3020.

11. Earth Microbiome Project. Illumina 16s PCR protocols. https://earthmicrobiome.org/protocols-andstandards/16s/. Accessed 29 June 2020.

12. Bolyen E, Rideout JR, Dillon MR, Bokulich NA, Abnet CC, Al-Ghalith GA, et al: Reproducible, interactive, scalable and extensible microbiome data science using QIIME 2. Nat Biotechnol. 2019;37(8):852857. 
13. Quast C, Pruesse E, Yilmaz P, Gerken J, Schweer T, Yarza P, et al: The SILVA ribosomal RNA gene database project: improved data processing and web-based tools. Nucleic Acids Res. 2013;41(Database issue):D590-D596.

14. ggplot2: Elegant Graphics for Data Analysis. https://ggplot2.tidyverse.org/. Accessed 29 June 2020.

15. Segata N, Izard J, Waldron L, Gevers D, Miropolsky L, Garrett WS, et al: Metagenomic biomarker discovery and explanation. Genome Biol. 2011;12(6):R60.

16. Langille MGI: Exploring Linkages between Taxonomic and Functional Profiles of the Human Microbiome. mSystems. 2018;3(2).

17. Parks DH, Tyson GW, Hugenholtz P, Beiko RG: STAMP: statistical analysis of taxonomic and functional profiles. Bioinformatics. 2014;30(21):3123-3124.

18. Storey JD: The positive false discovery rate: a Bayesian interpretation and the q-value. Ann Statist. 2003;31(6):2013-2035.

19. Wilson MJ, Dekker JWT, Harlaar JJ, Jeekel J, Schipperus M, Zwaginga JJ: The role of preoperative iron deficiency in colorectal cancer patients: prevalence and treatment. International Journal of Colorectal Disease. 2017;32(11):1617-1624.

20. Gereklioglu C, Asma S, Korur A, Erdogan F, Kut A: Medication adherence to oral iron therapy in patients with iron deficiency anemia. Pakistan Journal of Medical Sciences. 2016;32(3):604-607.

21. Brookes MJ, Boult J, Roberts K, Cooper BT, Hotchin NA, Matthews G, et al: A role for iron in Wnt signalling. Oncogene. 2008;27(7):966-975.

22. Radulescu S, Brookes MJ, Salgueiro P, Ridgway RA, McGhee E, Anderson K, et al: Luminal iron levels govern intestinal tumorigenesis after Apc loss in vivo. Cell Rep. 2012;2(2):270-282.

23. Chua ACG, Klopcic BRS, Ho DS, Fu SK, Forrest CH, Croft KD, et al: Dietary iron enhances colonic inflammation and IL-6/IL-11-Stat3 signaling promoting colonic tumor development in mice. PloS One. 2013;8(11):e78850.

24. Flemer B, Lynch DB, Brown JMR, Jeffery IB, Ryan FJ, Claesson MJ, et al: Tumour-associated and nontumour-associated microbiota in colorectal cancer. Gut. 2017;66(4):633-643.

25. Kim DJ, Yang J, Seo H, Lee WH, Ho Lee D, Kym S, et al: Colorectal cancer diagnostic model utilizing metagenomic and metabolomic data of stool microbial extracellular vesicles. Sci Rep. 2020;10.

26. Drago L: Probiotics and Colon Cancer. Microorganisms. 2019;7(3).

27. Ma Q, Li Y, Wang J, Li P, Duan Y, Dai H, et al: Investigation of gut microbiome changes in type 1 diabetic mellitus rats based on high-throughput sequencing. Biomedicine \& Pharmacotherapy. 2020;124:109873.

28. Li H, Liu F, Lu J, Shi J, Guan J, Yan F, et al: Probiotic Mixture of Lactobacillus plantarum Strains Improves Lipid Metabolism and Gut Microbiota Structure in High Fat Diet-Fed Mice. Front Microbiol. 2020;11.

29. Lee T, Clavel T, Smirnov K, Schmidt A, Lagkouvardos I, Walker A, et al: Oral versus intravenous iron replacement therapy distinctly alters the gut microbiota and metabolome in patients with IBD. Gut. 
2017;66(5):863-871.

30. Miethke M, Marahiel MA: Siderophore-Based Iron Acquisition and Pathogen Control. Microbiol Mol Biol Rev. 2007;71(3):413-451.

31. Rivera M: Bacterioferritin: Structure, Dynamics, and Protein-Protein Interactions at Play in Iron Storage and Mobilization. Acc Chem Res. 2017;50(2):331-340.

32. Torti SV, Torti FM: Iron and cancer: more ore to be mined. Nat Rev Cancer. 2013;13(5):342-355.

33. Di Costanzo L, Gomez GA, Christianson DW: Crystal Structure of Lactaldehyde Dehydrogenase from Escherichia coli and Inferences Regarding Substrate and Cofactor Specificity. J Mol Biol. 2007;366(2):481-493.

34. Iraporda C, Romanin DE, Bengoa AA, Errea AJ, Cayet D, Foligné B, et al: Local Treatment with Lactate Prevents Intestinal Inflammation in the TNBS-Induced Colitis Model. Front Immunol. 2016;7.

35. Algieri F, Rodriguez-Nogales A, Garrido-Mesa J, Camuesco D, Vezza T, Garrido-Mesa N, et al: Intestinal anti-inflammatory activity of calcium pyruvate in the TNBS model of rat colitis: Comparison with ethyl pyruvate. Biochem Pharmacol. 2016;103:53-63.

36. Murata K, Moriyama M: Isoleucine, an essential amino acid, prevents liver metastases of colon cancer by antiangiogenesis. Cancer Res. 2007;67(7):3263-3268.

37. Konno Y, Ashida T, Inaba Y, Ito T, Tanabe H, Maemoto A, et al: Isoleucine, an Essential Amino Acid, Induces the Expression of Human $\beta$ Defensin 2 through the Activation of the G-Protein Coupled Receptor-ERK Pathway in the Intestinal Epithelia. Food and Nutrition Sciences. 2012;3(4):548-555.

38. Kim Y, Hwang SW, Kim S, Lee Y, Kim T, Lee S, et al: Dietary cellulose prevents gut inflammation by modulating lipid metabolism and gut microbiota. Gut Microbes. 2020;11(4):944-961.

39. Chen H, Lin Y, Wang Y: Comparative Effects of Cellulose and Soluble Fibers (Pectin, Konjac Glucomannan, Inulin) on Fecal Water Toxicity toward Caco-2 Cells, Fecal Bacteria Enzymes, Bile Acid, and Short-Chain Fatty Acids. J Agric Food Chem. 2010;58(18):10277-10281.

40. Kantor ED, Lampe JW, Navarro SL, Song X, Milne GL, White E: Associations Between Glucosamine and Chondroitin Supplement Use and Biomarkers of Systemic Inflammation. J Altern Complement Med. 2014;20(6):479-485.

41. Kantor ED, Newton CC, Giovannucci EL, McCullough ML, Campbell PT, Jacobs EJ: Glucosamine Use and Risk of Colorectal Cancer: Results from the Cancer Prevention Study II Nutrition Cohort. Cancer Causes Control. 2018;29(3):389-397.

\section{Tables}

Table 1: Patient cohort demographics. Categorical variables are presented with percentages. Continuous variables are presented as mean value [standard deviation] or *median value [interquartile range]. $\mathrm{Hb}$, haemoglobin. CR-POSSUM, ColoRectal Physiological and Operative Severity Score for the enumeration of Mortality and morbidity. ASA, American Society of Anaesthesiologist. 


\begin{tabular}{|c|c|c|}
\hline & Oral Iron $(n=16)$ & Intravenous Iron $(n=24)$ \\
\hline Age & $74.9[7.4]$ & $74.9[9.5]$ \\
\hline Male & $8(50 \%)$ & $16(67 \%)$ \\
\hline Female & $8(50 \%)$ & $8(33 \%)$ \\
\hline Height, m & $1.66[0.1]$ & $1.70[0.9]$ \\
\hline Weight, kg & $72.7[17.4]$ & 79.3 [17.3] \\
\hline Inclusion $\mathrm{Hb}, \mathrm{g} / \mathrm{I}$ & $100.3[10.6]$ & $98.8[13.1]$ \\
\hline Recruitment ferritin, $\mu \mathrm{g} / \mathrm{l}$ * & $24.5[11.1-37.3]$ & $23[10-48.3]$ \\
\hline Recruitment transferrin saturation, \% * & $2.9[2.5-3.3]$ & $2.8[2.4-3.3]$ \\
\hline Duration of iron treatment, days * & $26.5[15-43]$ & $23.5[15-40.5]$ \\
\hline \multicolumn{3}{|l|}{ Tumour Features } \\
\hline Tumour size, mm & $48.5[25.3]$ & $42.4[23.2]$ \\
\hline \multicolumn{3}{|l|}{ Tumour Stage } \\
\hline $\mathrm{T} \leq 2$ & $1(6.25 \%)$ & $3(12.5 \%)$ \\
\hline T3 & $10(62.5 \%)$ & $18(75 \%)$ \\
\hline $\mathrm{T} 4$ & $5(31.25 \%)$ & $3(12.5 \%)$ \\
\hline \multicolumn{3}{|l|}{ Tumour Location } \\
\hline Right colon & $14(87.5 \%)$ & $17(71 \%)$ \\
\hline Left colon & $2(12.5 \%)$ & $7(29 \%)$ \\
\hline \multicolumn{3}{|l|}{ Preoperative Risk Assessment } \\
\hline \multicolumn{3}{|l|}{ ASA fitness status classification } \\
\hline I-II & $12(75 \%)$ & $10(42 \%)$ \\
\hline III-IV & 4 (25\%) & $14(58 \%)$ \\
\hline CR-POSSUM mortality score, \% * & $3.6[2.8-9.3]$ & $3.5[2.6-8.6]$ \\
\hline
\end{tabular}

\section{Figures}


(a)

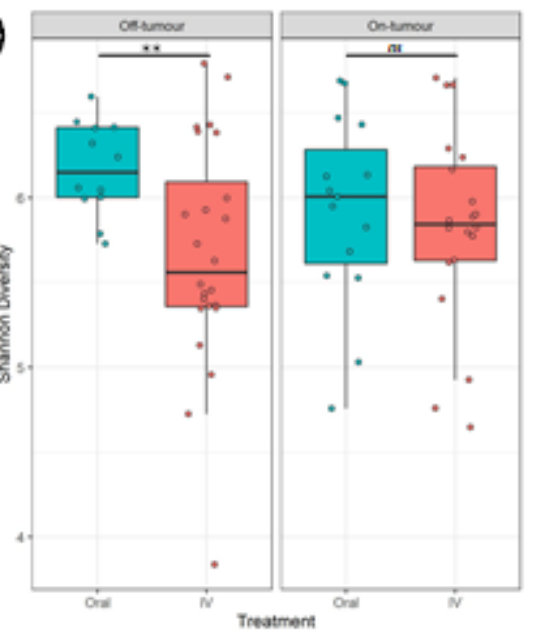

(b)

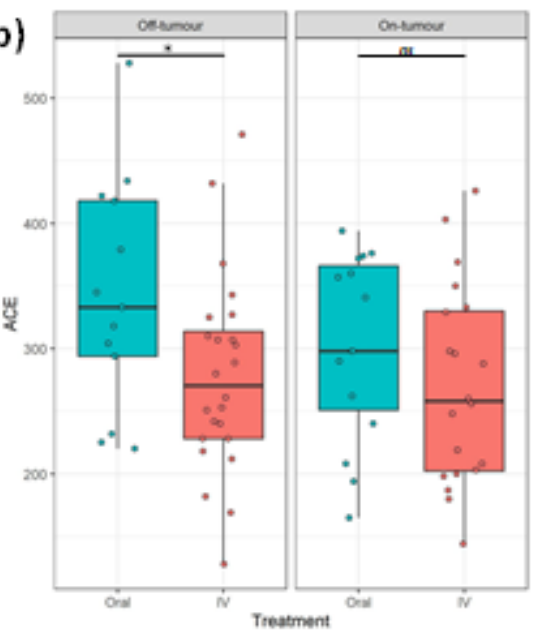

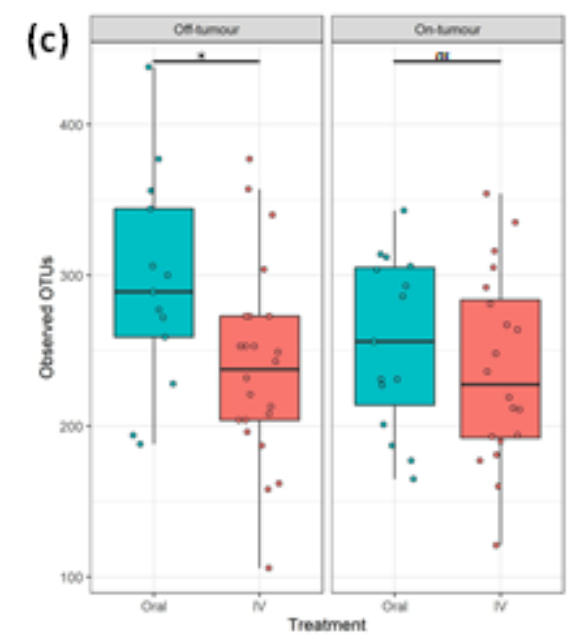
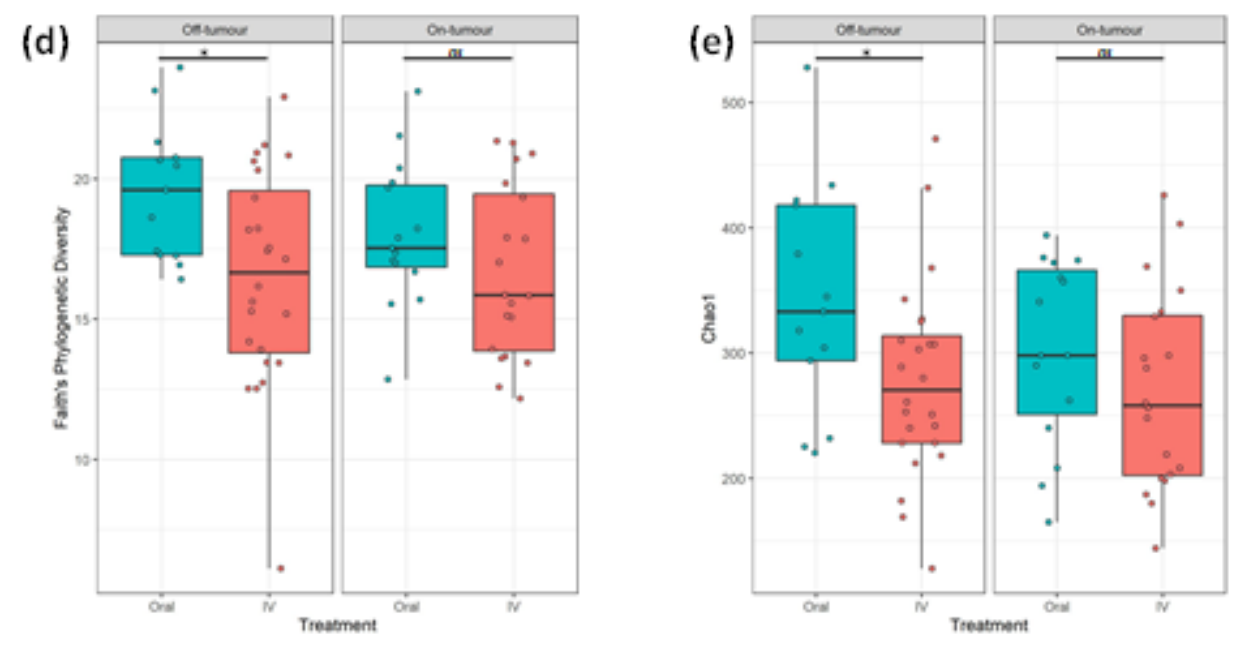

\section{Figure 1}

On- and off-tumour a-diversity is differentially altered following iron therapy. a-diversity metrics (a) Shannon Diversity, (b) Abundance-based coverage estimate (ACE), (c) Observed operational taxonomic units (OTUs), (d) Faith's Phylogenetic Diversity and (e) Chao1. Off-tumour diversity metrics were significantly greater in oral iron compared to intravenous (IV) treated patients. On-tumour diversity showed no significant differences between oral and IV iron-treated patients $\left(* p \leq 0.05,{ }^{*} p \leq 0.01\right.$, ns $p>0.05)$. 

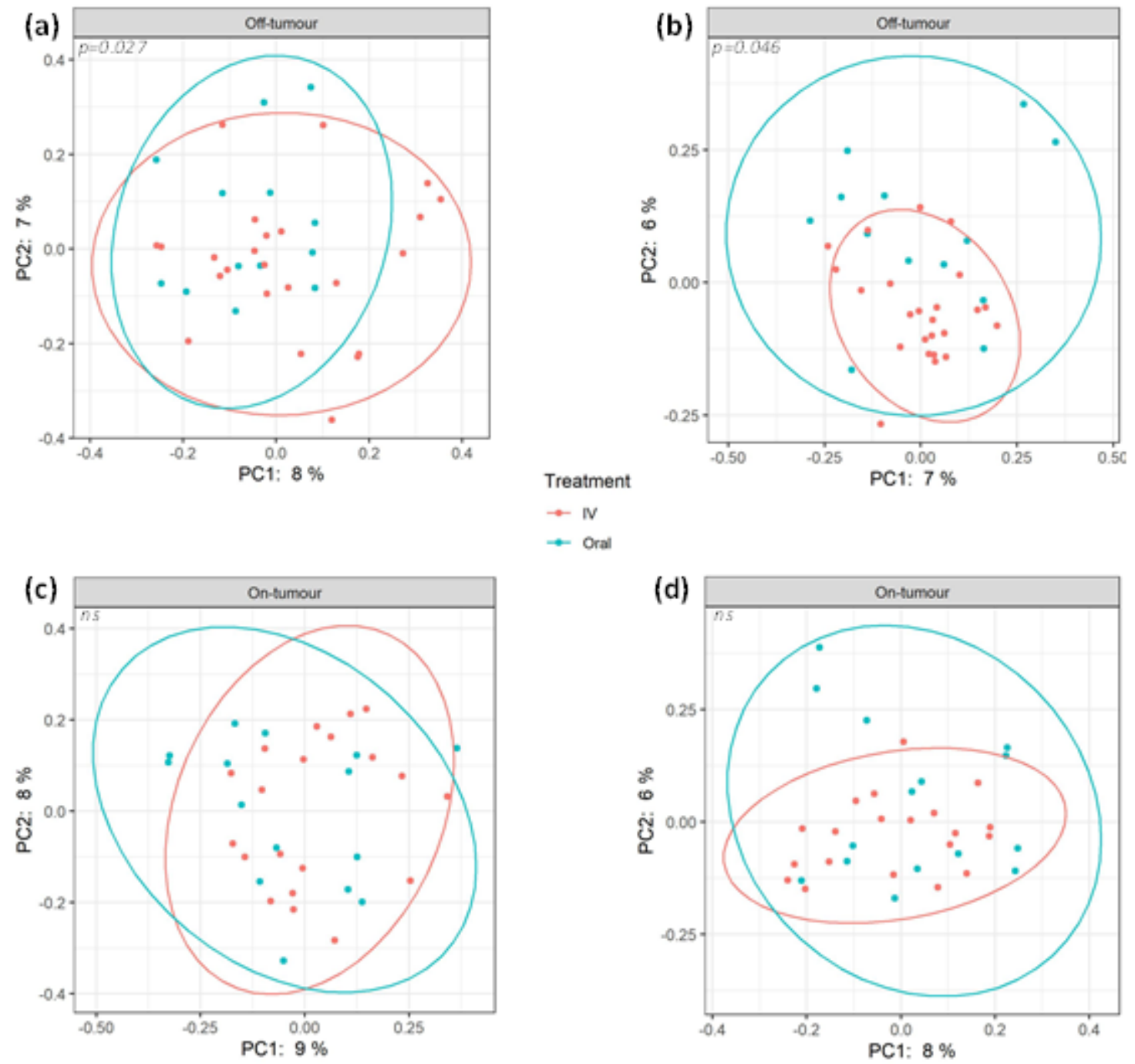

Figure 2

$\beta$-diversity of oral and intravenous iron-treated patients differs between on- and off-tumour microbiota. Principle coordinate analysis (PCoA) plots based on Bray-Curtis (a, c) and Jaccard (b, d) distances. Plot ellipses represent $95 \%$ confidence incidence for group clusters. The off-tumour $\beta$-diversity shows significantly distinct bacterial community clusters between oral and intravenous (IV) iron treatments (a; $p=0.027, b ; p=0.046$ ). On-tumour $\beta$-diversity shows no significant differences (ns) between iron treatment $(c, d)$. 
(a)

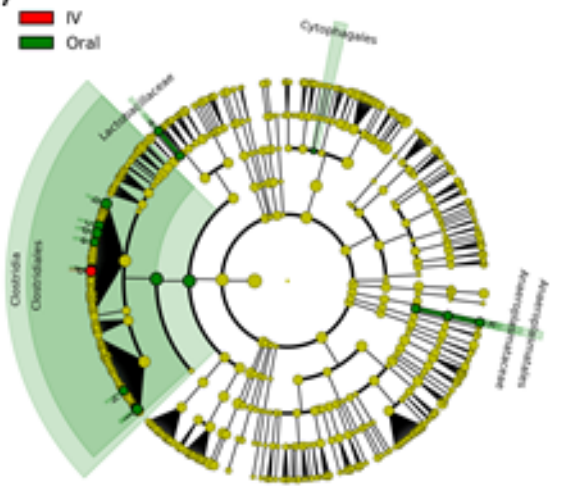

(c) $=\mathrm{Nat}_{\text {orat }}^{\mathrm{N}}$

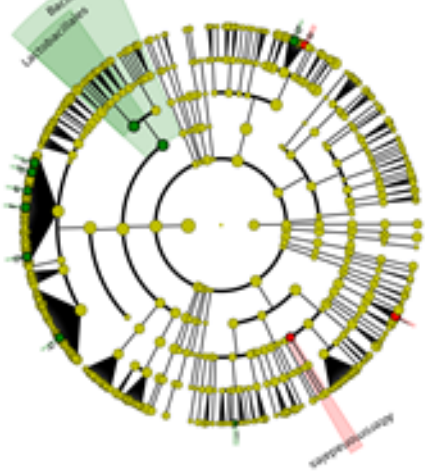

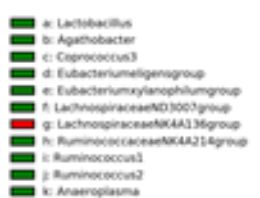

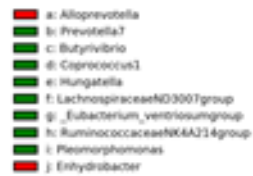

(d)

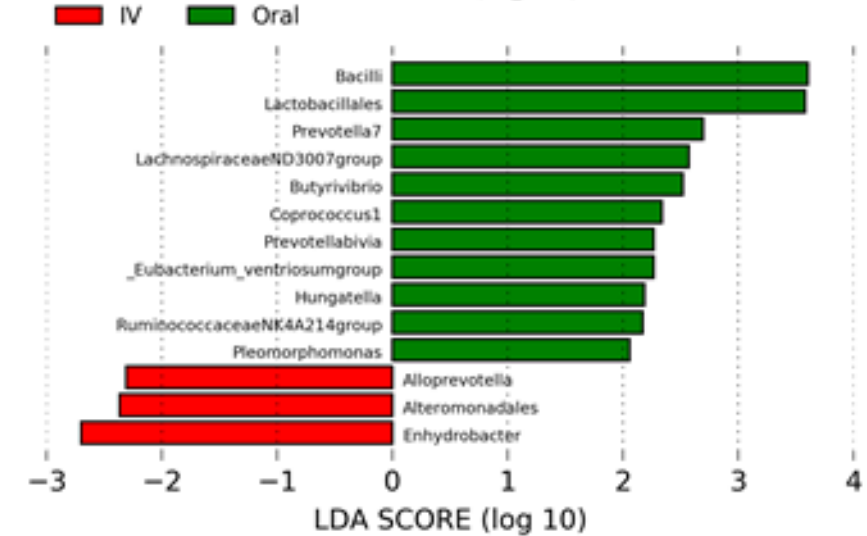

Figure 3

Linear discriminant analysis (LDA) effect size (LEfSe) comparing iron therapy on bacterial taxa in the onand off-tumour microbiota. Histograms of LDA scores for differentially abundant bacterial taxa between oral and intravenous (IV) iron-treated patients in off-tumour (b) and on-tumour (d) microbiota. LEfSe cladogram representing differentially abundant bacterial taxa in off-tumour (a) and on-tumour (c) microbiota between iron treatments. Differentially abundant taxa from phylum to genus taxonomic levels were included. Taxa and nodes highlighted in green were more abundant in oral and red in IV iron-treated patients. Taxa with an LDA $>2$ with a p-value $\leq 0.05$ were considered statistically significant. 
(a)

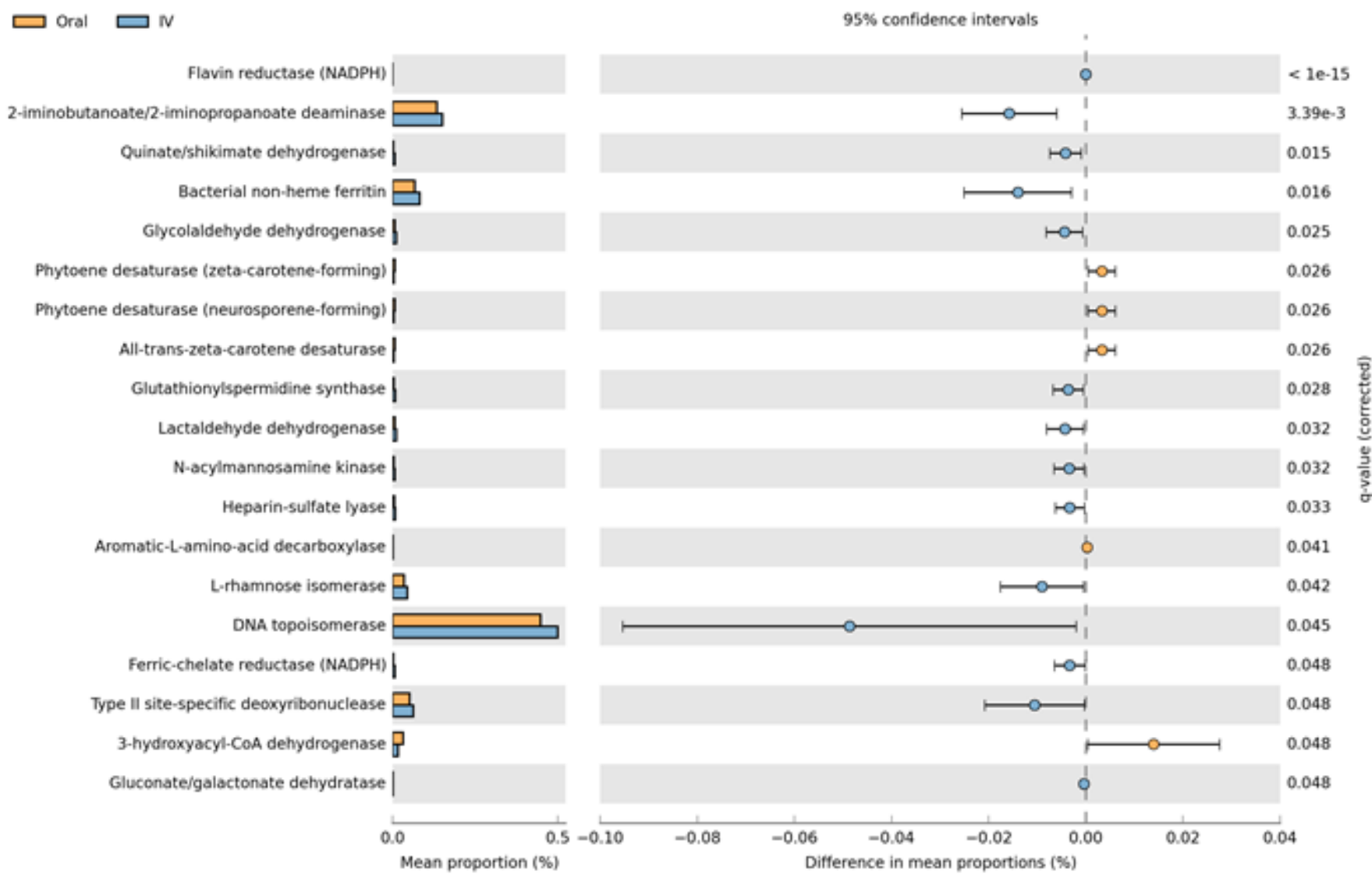

(b)

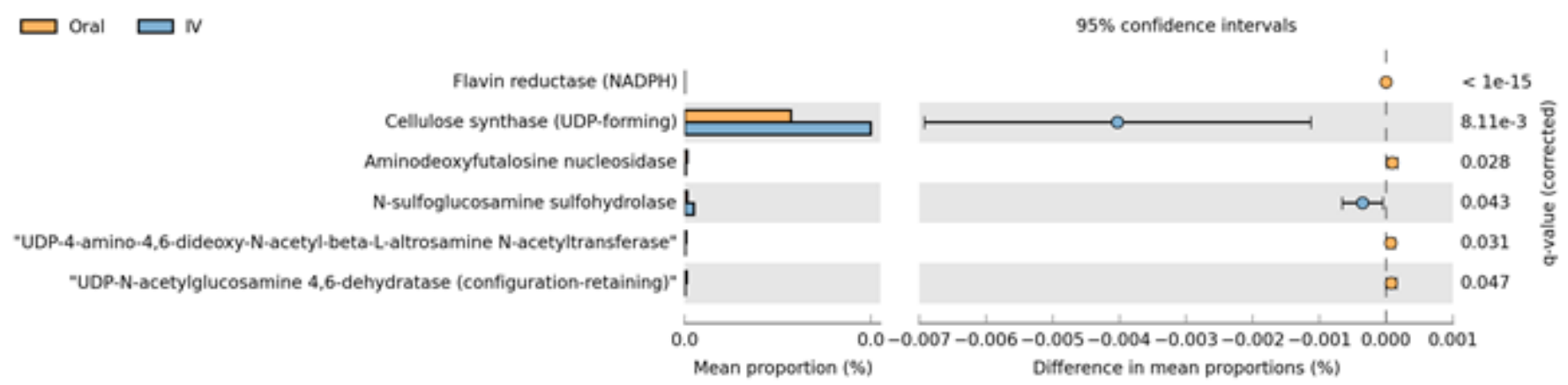

\section{Figure 4}

Comparison of predictive metagenomics between iron treatment groups. Predictive metagenomic analysis of enzyme classification abundances between oral and intravenous (IV) iron treatments, based on off-tumour (a) and on-tumour (b) microbial populations. Corrected q-values were calculated following multiple testing correction using Storey false discovery rate. q-values $\leq 0.05$ were considered statistically significant. 
(a)

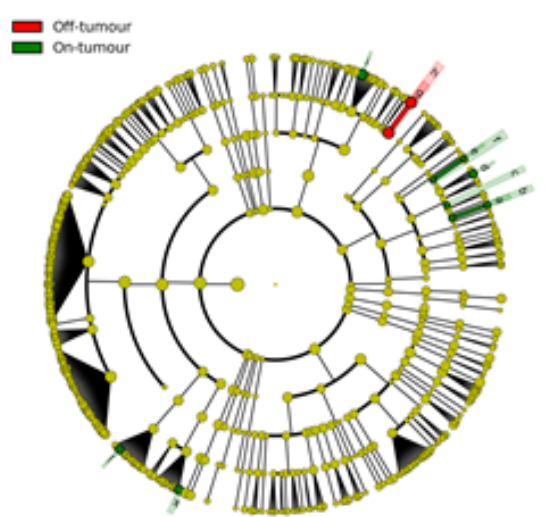

(c)

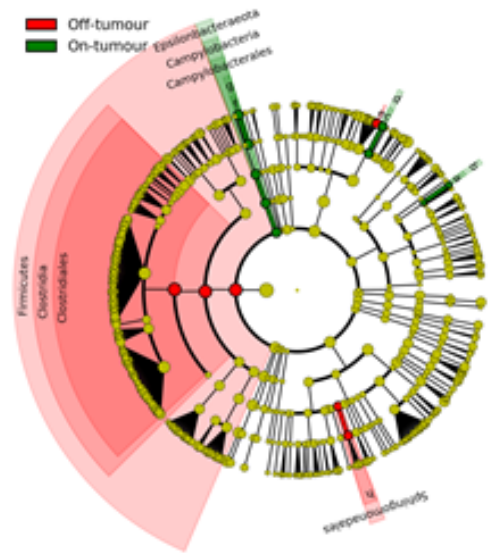

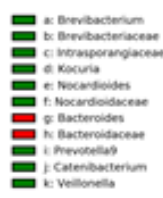

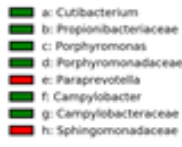

(b)

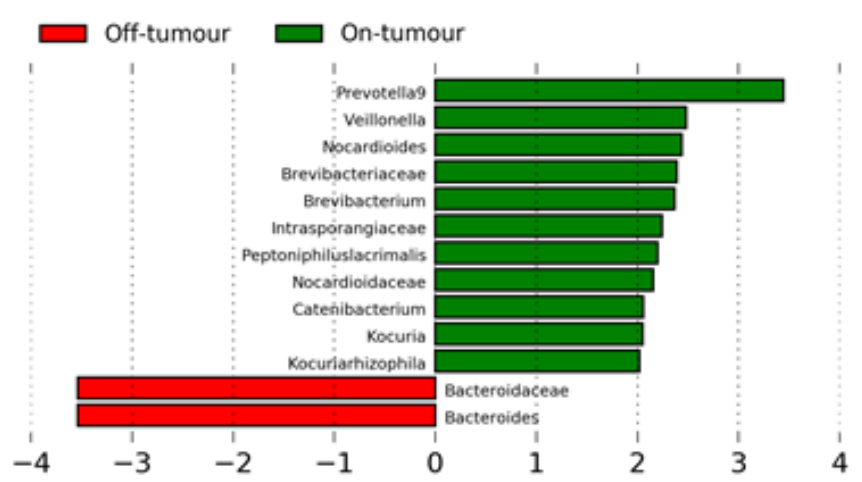

(d)

LDA SCORE $(\log 10)$

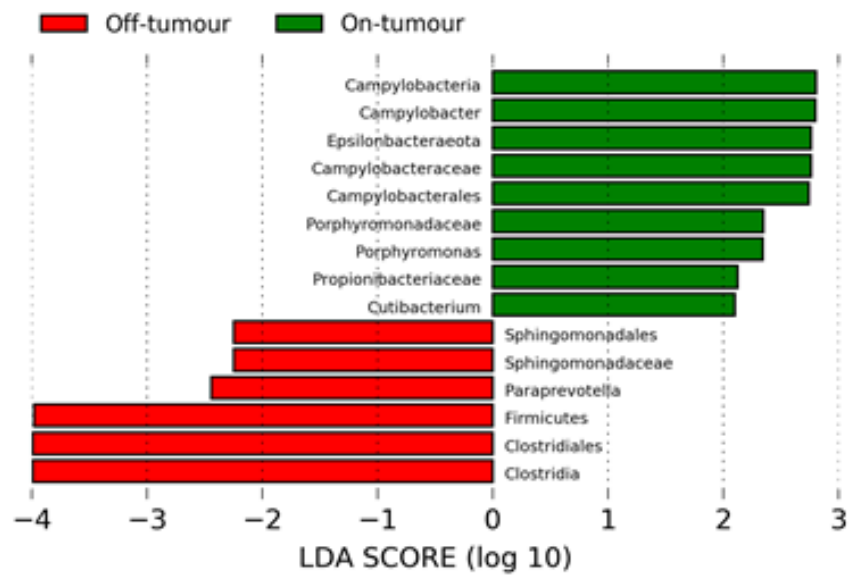

Figure 5

Paired comparison of on-and off-tumour taxa between treatment groups. LDA scores for differentially abundant bacterial taxa between on- and off-tumour microbiota in oral (b) and intravenous (d) irontreated patients. LEfSe cladogram demonstrating differential abundant bacterial taxa from genus to phylum taxonomic levels between paired on- and off-tumour microbiota in oral (a) and intravenous (c) iron-treated iron patients. Taxa and nodes highlighted in red were more significant in the off-tumour microbiota and green in the on-tumour microbiota. Taxa with an LDA score greater than 2 with a p-value $\leq 0.05$ were considered statistically significant. 